\title{
Neues über Beihilfen und zum
}

\section{Vergaberecht}

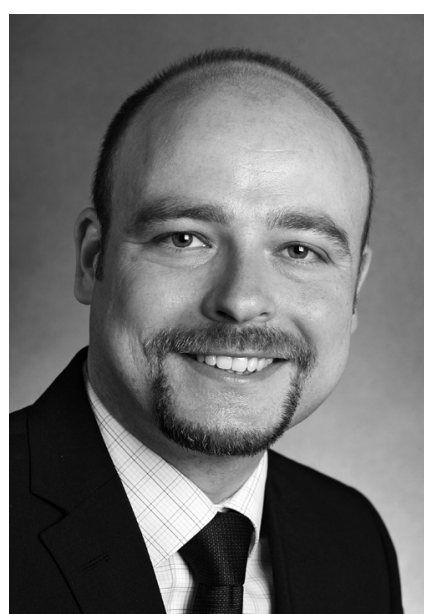

VON MICHAEL MÜLLER Michael Müller (LL.M. Eur.) ist Volljurist und arbeitet seit 2008 für die Hauptvertretung Brüssel des Deutschen Caritasverbandes, deren Leitung er 2011 übernahm. Ein Schwerpunkt seiner Arbeit liegt in Fragestellungen rund um die Auswirkungen des Europarechts auf die freigemeinnützige Erbringung sozialer Dienstleistungen in Deutschland.

michael.mueller@caritas.de

\author{
Neben der Sozialpolitik und den europäischen \\ Förderprogrammen sind das Binnenmarkt- und \\ Wettbewerbsrecht aus Sicht eines freigemeinnützigen \\ Sozialdienstleistungserbringers die zentralen \\ Interessenfelder der Politik der Europäischen Union \\ und des Europarechtes. In beiden Bereichen hat sich \\ in letzter Zeit einiges getan.
}

Für Unternehmensverantwortliche in der Sozial- und Gesundheitswirtschaft ist es heute geradezu unerlässlich, sich auch mit den Grundzügen von europäischem Beihilfe- und Vergaberecht auseinanderzusetzen. Die beiden zentralen Fragestellungen lauten dabei:

- Sind staatliche Zuwendungen für Dienstleistungserbringer europarechtskonform?

- Müssen soziale Dienstleistungen von Seiten der öffentlichen Hand europaweit ausgeschrieben werden?

\section{Beihilferecht}

Das EU-Beihilferecht ist Teil des Wettbewerbsrechts und wird für gemeinnützige Sozialleistungserbringer in Deutschland dort relevant, wo diese ihre Dienste im Wettbewerbsverhältnis mit anderen Anbietern erbringen. Ratio des Beihilferechts ist es dafür Sorge zu tragen, dass im gemeinsamen Binnenmarkt nicht einzelne Mitgliedstaaten protektionistische Maßnahmen zu Gunsten der einheimischen Wirtschaft ergreifen und damit Wettbewerber aus anderen Mitgliedstaaten diskriminieren.

Eine Befassung mit den entsprechenden Vorschriften ist für Unternehmensverantwortliche in der Freien Wohlfahrtspflege schon deshalb anzuraten, weil über allen Zuwendungen das Damoklesschwert der Rückforderung schwebt. Beihilfen, die sich im Zuge einer Überprüfung als eu- roparechtswidrig herausstellen, sind vom Zuwendungsempfänger zurückzuerstatten. Diese strikte und mitunter für das betroffene Unternehmen ruinöse Folge gilt es durch vorausschauende Beachtung der relevanten Vorschriften zu vermeiden.

Kern der Vorschriften ist die Frage, in welchem Umfang öffentliche Mittel zur Finanzierung von im Wettbewerb stehenden Marktteilnehmern herangezogen werden können. Dabei geht das Europarecht in Art. 107 Abs. 1 des Vertrags über die Arbeitsweise der Europäischen Union (AEUV) davon aus, dass derartige geldwerte Unterstützungsleistungen mit dem Binnenmarkt unvereinbar sind, soweit sie den Wettbewerb verfälschen oder zu verfälschen drohen. Es gibt also ein grundsätzliches Beihilfeverbot im Europarecht. Beihilfen in diesem Sinne sind im Übrigen nicht lediglich Subventionen im engeren Sinne, sondern alle geldwerten Vorteile, denen keine marktübliche Gegenleistung gegenübersteht (Steuerermäßigungen, Steuererlasse, zinslose Darlehen, Bürgschaften etc.).

Gleichzeitig anerkennen auch die EUVerträge, dass dem Erfordernis eines fairen Wettbewerbs auf dem Binnenmarkt die Notwendigkeit einer stabilen und nachhaltigen Finanzierung von sozialen Dienstleistungen in den Mitgliedstaaten gegenübersteht. Aus diesem Grunde lässt Art. 106 Abs. 2 AEUV für soziale und andere Daseinsvorsorgedienste gewisse Ausnahmen vom Wettbewerbsrecht zu. Genau diese Ausnahmevorschriften wur- 
den im Jahr 2012 im »Almunia-Paket", benannt nach EU-Wettbewerbskommissar Joaquín Almunia, reformiert.

Das Reformpaket besteht aus mehreren Bestandteilen, von denen für die Freie Wohlfahrtspflege vor allem der Freistellungsbeschluss (1), die De-minimis-Verordnung (2) sowie die Mitteilung (3) der Kommission von Interesse sind. Nicht im Paket enthalten war die Überarbeitung der Allgemeinen Gruppenfreistellungsverordnung (AGVO) (4). Diese soll noch im Laufe des Jahres 2014 zum Abschluss gebracht werden.

Im Ergebnis lässt sich festhalten, dass die Kommission ihrem mit der Reform selbstgesteckten Ziel der Vereinfachung der Vorschriften nur teilweise gerecht wurde. Positiv ist sicherlich zu werten, dass mit der neuen De-minimis-Verordnung für den Bereich der Daseinsvorsorge eine langjährige Forderung der Freien Wohlfahrtspflege umgesetzt wurde. Zuwendungen, die unter der De-minimisSchwelle liegen, sind mangels Relevanz für den innergemeinschaftlichen Handel vom Beihilferecht ausgenommen. Gleichwohl bietet auch diese neue De-minimisRegel mit ihrem Schwellenwert von 500.000 Euro (Höhe aller Zuwendungen an ein Unternehmen in drei Steuerjahren) allenfalls für kleine Zuwendungsempfänger einen Ausweg aus dem Beihilferecht.

Alle Unternehmen, die wegen Überschreiten des Schwellenwertes nicht unter die De-minimis-Regel fallen, bleibt in der Regel lediglich der Weg über den Freistellungsbeschluss. Dessen Rechtsfolge ist, dass es sich bei den betroffenen $\mathrm{Zu}$ wendungen zwar um Beihilfen im Sinne des Art. 107 Abs. 1 AEUV handelt, diese aber mit dem Binnenmarkt vereinbar sind und nicht bei der Kommission notifiziert werden müssen. Für die beihilferechtliche Würdigung der jeweiligen Zuwendung gelten dabei die bekannten Kriterien des Urteils des Europäischen Gerichtshofes in der Rechtssache Altmark-Trans (5).

Nach wie vor offen ist leider die beihilferechtliche Behandlung der Steuervorteile aus der Gemeinnützigkeit. Hier$\mathrm{zu}$ hat die Kommission in der Reform keine Aussage getroffen. Es gibt zwar gute Argumente, die dafür sprechen die Steuervorteile nicht als Beihilfe im Sinne des Vertrags über die Arbeitsweise der Europäischen Union zu werten, Rechtssicherheit besteht bis zu einem klärenden Urteil des Europäischen Gerichtshofes aber nicht.
Der Deutsche Caritasverband vertritt die Auffassung, dass sich gemeinnützige Unternehmen aufgrund der zahlreichen mit der Gemeinnützigkeit einhergehenden Einschränkungen der unternehmerischen Handlungsfreiheit nicht in einer tatsächlich und rechtlich vergleichbaren Situation mit gewinnorientierten Unternehmen befinden. Dies wäre aber Voraussetzung für das Vorliegen einer Beihilfe im Sinne des Europarechtes. Die Kommission hat sich dieser Argumentation in einem jüngeren Beschwerdeverfahren angeschlossen, allerdings nur für den betroffenen Einzelfall ohne präjudizielle Wirkung für etwaige Folgeverfahren.

\section{Vergaberecht}

Die Modernisierung des europäischen Vergaberechts wurde Anfang 2014 abgeschlossen. Mit der Veröffentlichung der Vorschriften im Amtsblatt der Europäischen Union haben die Mitgliedstaaten zwei Jahre Zeit die Vorgaben aus Brüssel in nationales Recht umzusetzen. Aus Sicht der Freien Wohlfahrtspflege sind die Ergebnisse der Reform überwiegend zu begrüßen.

Als sicherlich wichtigster Lobbyerfolg konnte erreicht werden, dass die neuen Richtlinien zur Vergabe öffentlicher Aufträge sowie von Dienstleistungskonzessionen nicht dazu geführt haben, dass die im deutschen Sozialrecht vorherrschende Leistungserbringung im klassischen sozialrechtlichen Dreiecksverhältnis künftig formellen Vergabeverfahren unterworfen wird. Beide Richtlinien stellen nämlich in jeweils einem Erwägungsgrund klar, dass Verfahren, bei denen wie im sozialrechtlichen Dreiecksverhältnis alle Anbieter, die die gesetzlichen Voraussetzungen erfüllen zur Leistungserbringung zugelassen werden als reine Zulassungsverfahren nicht in den Anwendungsbereich der Richtlinien und damit auch nicht unter formelle Vergabeverfahren fallen. In Art. 1 Abs. 2 der Richtlinie über die Auftragsvergabe wird zudem nun ausdrücklich auf eine Auswahlentscheidung des Auftraggebers Bezug genommen. Liegt diese, etwa weil es einen allgemeinen Zulassungsanspruch gibt, nicht vor, ist der Anwendungsbereich der Richtlinie nicht eröffnet. Diese Klarstellung dürfte künftig nationalen Versuchen mit Verweis auf vermeintliche europarechtliche Vorgaben das sozialrechtliche Dreiecksverhältnis dem Vergaberecht zu unterwerfen einen Riegel vorschieben.
Sehr erfreulich ist auch, dass der Rettungsdienst (anders als der Krankentransport), soweit er von gemeinnützigen Organisationen ausgeübt wird, nicht in den Anwendungsbereich der Richtlinie fällt.

Soweit die Vergabe von Sozial-, Gesundheits- oder Arbeitsmarktdienstleistungen (außerhalb des sozialrechtlichen Dreiecksverhältnisses) aber in den Anwendungsbereich der Richtlinie fällt, gilt für diese ein erhöhter Schwellenwert von 750.000 Euro sowie ein erleichtertes Vergaberegime. Dieses lässt den Mitgliedstaaten einen großen Ermessenspielraum, um für diese besonderen Dienstleistungen geeignete Verfahren zu entwickeln. Festgeschrieben wurden allerdings gewisse, von den Mitgliedstaaten zu achtende Grundsätze, wie etwa die Qualität, Kontinuität, Bezahlbarkeit und Zugänglichkeit der Dienstleistungen sowie das Erfordernis den besonderen Bedürfnissen benachteiligter und schutzbedürftiger Nutzer gerecht zu werden. Aus Sicht der Freien Wohlfahrtspflege kommt es nun entscheidend darauf an, dass der Bundesgesetzgeber den Ball der EU-Kommission aufgreift. Die Verbände bieten der Bundesregierung ihre Erfahrungen im Umgang mit Ausschreibungsverfahren im Bereich sozialer Dienstleistungen an. Ziel der nationalen Umsetzung der Richtlinien muss es sein, auf Grundlage der europäischen Vorgaben ein deutsches Sozialvergaberecht zu entwickeln, das den Bedürfnissen der Nutzer entspricht und deren Wunsch- und Wahlrecht achtet und nicht den günstigsten Preis eines Anbieters zum entscheidenden Kriterium für den Zuschlag macht.

\section{Anmerkungen}

(1) Beschluss der Kommission, 2012/21/EU, ABl. L 7, S. 3.

(2) Verordnung Nr. 360/2012, ABl. L 114, S. 8.

(3) Mitteilung der Kommission vom 20. Dezember 2011, K(2011), 9404 endgültig.

(4) Verordnung EG Nr. 800/2008, ABl. L 214, S. 3.

(5) EuGH, Rs. C-280/00, Slg. 2003, I-7747; dabei ging es um Zuschüsse zur Erbringung von öffentlichen Personennahverkehrs-Dienstleistungen, die die Mehrkosten ausgleichen sollten, die durch die Bedienung wirtschaftlich unrentabler Strecken entstanden. 